\title{
PERCEPTION OF (THE) PSYCHOLOGICAL MOTIVES OF TATTOOING OF ALL AGES
}

\author{
Anna Winiarek ${ }^{1}, \&$ Ludwika Wojciechowska ${ }^{2}$ \\ ${ }^{1}$ Polish Academy of Science, Institute of Psychology (Poland) \\ ${ }^{2}$ University of Zielona Góra, Faculty of Education, Psychology and Sociology (Poland)
}

\begin{abstract}
Reviewing the current psychological research, one can reach a conclusion that previously tattooing the body has been mainly seen through the perspective of clinical psychology - usually as body self-mutilation, proving a negative attitude to oneself, or as the occurrence of mental disorders. Tattooing yourself was also often associated with other risk-taking behaviors such as the use of psychoactive substances.

Currently, more and more people are tattooing themselves, more and more tattoo studios are being set up. Therefore, the idea was to look again at the motives of people's tattoos and how tattoos are currently perceived by people of all ages.

The study is question, which was carried out from October 2018 till April 2019, focuses on the perception of the motives of tattooing trough different age cohorts.

A total of 407 people from different development stage (pre-school period, early school-age period, adolescents, early adults, middle adults and late adults) took part in the study. The study was conducted using semi-structured interview.

The collected data were analyzed using the $x^{2}$ test, the $V$-Cramer test and with the Bonferroni method.

The results of the research have shown that people of different ages exhibit a different perspective of the motives for tattooing.

In addition, it turns out that the motives of tattooing are perceived not only as a negative, but also as a positive phenomenon, for example, indicating personal development and the pursuit of self-realization in accordance with assumption of Abraham Maslow's hierarchy of needs.

Detailed results will be presented during the conference.

The results obtained in the study will be used to create a standardized questionnaire to study the psychological motives of tattooing and another body modifications. The study is also the first stage of a broad research project on the mechanisms of body modifications among people of all ages.
\end{abstract}

Keywords: Development psychology, social psychology, personal growth, body modification, tattooing. 